\title{
Algorithmic Problem Solving - Three Years On
}

\author{
Roland Backhouse \\ School of Computer Science and Information Technology \\ University of Nottingham \\ Nottingham NG8 1BB, \\ England \\ rcb@cs.nott.ac.uk
}

\begin{abstract}
"Algorithmic problem solving" introduces problem-solving skills based on the principles of correct-by-construction algorithm design. Introduced three years ago as a 1st-year option, the module has overcome initial opposition, and became compulsory for all 1st-year students of Computer Science and Mathematics and Computer Science at the University of Nottingham from September, 2006. This paper gives a short introduction to the goals of the module and how the goals are being realised.
\end{abstract}

Keywords: algorithm, problem-solving, program construction

\section{INTRODUCTION}

The module entitled "algorithmic problem solving" (APS for short) was first offered as a 1st-year option in September 2003. In the three years that it has been given, it has steadily grown in popularity, in 2005-2006 attracting slightly more students than the hitherto most popular option "Introduction to Artificial Intelligence". In the current academic year, it became compulsory for all 1st-year students of Computer Science and Mathematics and Computer Science at the University of Nottingham.

The title of the module is deliberately ambiguous. Parsed as algorithmic-problem solving, it is about solving problems that involve the construction of an algorithm for their solution. Parsed as algorithmic problem-solving, it is about problem solving in general, using the principles that have been learnt in the development of correct-by-construction algorithm-design techniques [11].

This paper gives a brief account of the main elements of the module. Full details can be obtained by downloading the lecture material from the author's website (www.cs.nott.ac.uk/ $\mathrm{rcb}$ ). The paper concludes with an assessment of the success of the module.

\section{GOALS}

In order to properly explain the goals of the module, this section introduces the module as it is introduced to the students in the first lecture - via a problem and its solution ${ }^{1}$.

Knockout Tournament A knockout tournament is a series of games. Two players compete in each game; the loser is "knocked out" (i.e. doesn't play anymore), the winner carries on. The winner of the tournament is the player that is left after all other players have been knocked out. Suppose there are 1234 players in a tournament. How many games are played before the tournament winner is decided?

\footnotetext{
${ }^{1}$ The problems that are chosen vary from year to year. The problem chosen here is typical of the initial problem presented to the students, rather than being the initial problem. The discussion is also abbreviated for the purposes of this paper.
} 
Solution We are required to determine the number of games that have been played when the number of players has been reduced to one. Let us introduce two variables $g$ and $p$, denoting respectively the number of games that have been played and the number of players.

Initially, $g$ is 0 and $p$ is 1234 . Whenever a game is played, $g$ is increased by 1 and $p$ is decreased by 1 . This is expressed in computer-programming terms by the assignment statement

$$
g, p:=g+1, p-1 .
$$

Now, we are required to determine $g$ when $p$ has the value 1 . This is done by observing that the value of $g+p$ is unchanged by the assignment. It is called an invariant of the assignment. Since $g+p$ is initially $0+1234$, it will always be 1234 . In particular, when $p$ is $1, g+p$ will still be 1234 and, so, $g$ will then be 1233 . The total number of games played is thus 1233 , i.e. one less than the number of players. End of Solution

Even though this is a very simple problem, it illustrates well the modus operandi throughout the module:

- A problem is introduced which the students can readily comprehend in the course of the lecture itself.

- The problem is used to introduce a programming statement, in this case the assignment statement.

- Simultaneously with introducing the programming statement, the students are shown how to reason about the statement, in this case how to determine an invariant of the assignment. (In fact, the students themselves are invited to suggest a combination of $g$ and $p$ that is unchanged by the assignment, which they do.)

The module is, first and foremost, problem-oriented. The goal is to teach problem-solving skills that have become vital to correct-by-construction algorithm design. The problems have been carefully chosen to introduce these skills in a systematic fashion. It is no accident, for example, that the notion of an invariant is introduced in the very first example. (Note, however, that the term "invariant" is applied to an integer value, $g+p$, rather than to a boolean value, $g+p=1234$, in order to avoid unnecessary complexity.)

The chosen problems are typically challenging but easy to formulate in everyday English. They are thus deliberately not "mathematical", but they are used to demonstrate how mathematics (in Dijkstra's sense of the art of effective reasoning) is vital to their successful solution.

Programming elements introduced in turn are (multiple) assignments, pre- and postconditions, sequential decomposition, case analysis and induction. Elements of calculational logic $[8,1]$ are introduced early, and used throughout the module. The notation used for program statements is that of the guarded command language [5], one reason being that nondeterminism is an essential element of some of the problems.

Because of its introductory nature, the module is not about algorithm development. In particular, loops in their full generality are not discussed. An important decision made when designing the module was not to try to push the students too far; if problem solving is to be taught effectively, it is important that students are given sufficient time to tackle the problems for themselves, and the syllabus needs to be tailored accordingly.

\section{TOPICS}

In this section, we give a brief outline of some of the topics discussed in the module, in the order that they are presented. 


\subsection{Assertions}

The use of assertions, in particular pre- and postconditions, is introduced via river-crossing problems. These problems involve getting a number of people or things across a river under given constraints. Standard presentations use brute force - a very ineffective problem-solving technique- , and commit the sin of unnecessary naming (eg. the goat-cabbage-wolf problem in [10]). Beautiful solutions can be constructed by exploiting the fact that crossing a river is symmetric about the left and right banks; solutions are found by decomposing the problems into three parts, the first and last being "mirror images". An example is the so-called "jealous-couples" problem (also known as the the "presidents-and-bodyguards" problem).

In the jealous-couples problem, there are three "couples" each consisting of a "husband" and "wife". There is one boat, which can take no more than two people at once. It is required to get everyone across the river under the constraint that a wife cannot be with a husband unless her own husband is also present.

A first step is to introduce notation. A state is denoted by two sequences separated by bars. An example is $3 H \| 3 W$, which denotes the state in which all three husbands are at the left bank, and all three wives are at the right bank. A second example of a state is $1 C, 2 H \| 2 W$, which denotes the state in which one couple and two husbands are at the left bank and two wives are at the right bank. The starting state is thus $3 C \|$ and the required finishing state is $\| 3 C$.

An action (or state transition) is when some individuals are being transported across the river. An example is $3 H|2 W| 1 W$; this denotes the action of transporting two wives across the river, leaving three husbands at the left bank and one wife at the right bank.

Having introduced notation, the goal, as well as a strategy for its achievement, can be formulated precisely. The goal is to construct a sequence of actions $S_{0}$ satisfying

$$
\{3 C \|\} \quad S_{0} \quad\{\| 3 C\} .
$$

Exploiting the left-right symmetry, one strategy is to decompose $S_{0}$ into $S_{1} ; S_{2} ; S_{3}$ where the three components are specified by:

$$
\begin{aligned}
& \{3 C \|\} \quad S_{1} \quad\{3 H \| 3 W\}, \\
& \{3 H \| 3 W\} \quad S_{2} \quad\{3 W \| 3 H\}, \\
& \{3 W \| 3 H\} \quad S_{3} \quad\{\| 3 C\} .
\end{aligned}
$$

The sequence $S_{1}$ changes the state from the start state to the state where all the wives are at the right bank and all the husbands are at the left bank. The function of $S_{3}$ is the mirror image, and $S_{2}$ provides the link between $S_{1}$ and $S_{3}$.

The remaining details are not relevant here. What is relevant is the problem-oriented way that sequential decomposition - specified formally in terms of pre- and postconditions- is introduced. Note particularly that, although the specification of the component actions is completely formal, a self-explanatory, domain-specific notation is used. Many introductions to "formal methods" view the goal as teaching some specific specification language, like Z or B or VDM, but this misses the real point. We should never forget that "formal methods" are only a tool to assist in problem solving and not an end in themselves.

\subsection{Games}

Simple impartial, two-person combinatorial games [3] form a significant part of the module. They fit the bill very well. After all, playing games is all about winning, which means determining an algorithm (the winning strategy), and the students need no motivation. Games are used in the module to introduce non-determinism and case analysis, as well as simple complexity issues and 
the idea of the development of a mathematical theory. The use of invariants —stressed from the outset of the module- is, of course, crucial.

For the class of games considered in the module, all positions can be categorised as winning or losing positions. A winning position is one from which there is a move to a losing position, and a losing position is one from which every move is to a winning position. Determining winning and losing positions thus affords an interesting exercise in the use of existential and universal quantification. The determination of a winning strategy is identified with an invariant: a property of positions such that, from a position that satisfies the property there is a move to a position that truthifies the property, and from a position that does not satisfy the property, every move is to one that falsifies the property.

For example, take a simple matchstick game. There is one pile of matches, and the two players take it in turns to remove some number $m$ of matches, where $m$ is at least 1 and at most $N$. The player who cannot move is the loser. The "invariant" is the property $k \bmod (N+1)=0$, where $k$ is the number of matches remaining. From a position satisfying this property every move falsifies the property, and from a position that does not satisfy the property, the removal of $k \bmod (N+1)$ matches truthifies the property (and is legal). The winning strategy is thus to truthify the property, if possible.

A highlight of the study of impartial games is the analysis of "sum" games. A "sum" game is a composite of two games. A position in the "sum" game is a pair of positions, one in each of the component games. A move in the "sum" game is determined by choosing one of the component games and moving in that game. (So, only one of the component positions is changed by a move.) Play is lost when it is not possible to move in either of the component games.

It is possible to form quite "difficult" games (in terms of the ease of identification of a winning strategy) by combining two very "simple" games. For example, the well-known game of Nim [4] is the "sum" of three copies of a trivial matchstick game (the game with one pile of matches from which any positive number of matches can be removed). More entertaining examples are constructed by "summing" two quite different, but nevertheless trivial, games. (The possibilities are endless, which is very useful for setting coursework and/or examination questions.)

A winning strategy for "sum" games is to maintain "symmetry" between the component games. Calling the component games the left and right games, two functions, $L$ and $R$ say, are defined on left and right positions, respectively, in such a way that a position $(l, r)$ is a losing position exactly when $L . l=R . r$. The question is: what properties should these functions satisfy? In other words, how do we specify the functions $L$ and $R$ ?

The analysis given earlier of a winning strategy allows one to distill the specification. First, since $(l, r)$ is an end position ${ }^{2}$ of the sum game exactly when $l$ is an end position of the left game and $r$ is an end position of the right game, it must be the case that $L$ and $R$ have equal values on end positions.

Second, every allowed move from a losing position - a position $(l, r)$ satisfying $L . l=R . r-$, that is not an end position, should result in a winning position - a position $(l, r)$ satisfying $L . l \neq R . r-$. That is,

$$
\begin{aligned}
& \{L . l=R . r \wedge(l \text { is not an end position } \vee r \text { is not an end position })\} \\
& \text { if } l \text { is not an end position } \rightarrow \text { change } l \\
& \square \quad r \text { is not an end position } \rightarrow \text { change } r \\
& \text { fi } \\
& \{L . l \neq R . r\} .
\end{aligned}
$$

${ }^{2}$ End positions are positions from which no move is possible; these are, by definition, losing positions. 
Third, applying the winning strategy, from a winning position - a position $(l, r)$ satisfying $L . l \neq R . r$ - should result in a losing position - a position $(l, r)$ satisfying $L . l=R . r-$. That is,

$$
\begin{aligned}
& \{L . l \neq R . r\} \\
& \text { apply winning strategy } \\
& \{L . l=R . r\} .
\end{aligned}
$$

This analysis of the requirements on $L$ and $R$ leads to the well-known "mex" (also called SpragueGrundy [9]) numbering of positions.

In the module, the above theory is used to develop the students' understanding of program specifications, and is reinforced by coursework. (The pace at which it is presented is, however, much, much slower!) Undoubtedly, they find it challenging but evidence provided by coursework and examination questions suggests that they find it rewarding.

\subsection{Induction}

As mentioned earlier, the module is not about the design of algorithms. It is very important that the students are allowed to develop confidence in their own problem-solving ability, and space must be allowed in the syllabus for that purpose. An unfortunate consequence is the omission of the design of loops in their full generality (maintaining an invariant whilst making progress towards the required postcondition). However, induction does form an important element of the module.

A major difference with standard accounts of induction is the stress on inductive construction rather than verification. This is an important point. Although I am myself an author of a book with "verification" in the title, I believe that the overwhelming emphasis on program "verification" in the formal-methods community is having a major detrimental effect on the adoption of formal methods.

There is an abundance of constructions that can be used to introduce the idea of induction in a much more convincing way than standard mathematical verifications (for example, of the formula for the sum of the first $n$ natural numbers). An inductive construction solves a problem by identifying the "size" of instances of the problem; it is then shown how to solve problems of size 0 - the base case - and how to solve problems of size $n+1$ assuming that problems of size at most $n$ can be solved. The problems I use in the module include the fake-coin problem [6,7], the Towers of Hanoi problem, and the Knight's-circuit problem. (The Towers of Hanoi problem illustrates how much our problem-solving ability has improved. Standard presentations of its solution are unnecessarily complex. The solution presented in APS, based on [2], exploits rotational symmetry of the poles and shows how invariant properties enable an iterative solution to be derived from the inductive solution.)

\section{CONCLUSIONS}

Although the problems presented in the Algorithmic Problem Solving (APS) course covered in this paper are mostly well-known (one might even say old-hat!) the "algorithmic" approach to their solution is very novel, indeed quite revolutionary. The success of the module is demonstrated by its increased popularity among students, and the fact that it has overcome initial opposition by academic staff. (It has become a compulsory module, albeit not for all the undergraduate degrees offered by the School, in spite of vigorous opposition to its introduction as an optional module three years ago.)

The most important factors contributing to its success are, I believe:

\section{- Avoidance of demotivation.}

There is often much discussion about how to "motivate" students. But this is the wrong question. My assumption is that students are motivated, but may lack self-confidence. They appreciate and are ready for the challenges of a university education, but may be unsure of 
how they will cope in a new environment. The biggest mistake we can make is to demotivate the students by not challenging them sufficiently, or by undermining their self-confidence by setting challenges that are unclear or beyond their grasp. The problems in APS are carefully chosen to achieve the right balance. They are challenging but doable. Most importantly, the students are able to appreciate how the methods taught in the module can improve their problem-solving skills.

\section{- Practical relevance.}

Problem-solving skills are generally acknowledged to be a vital element of undergraduate education. Algorithmic problem solving is a particularly important skill, since the formulation of algorithms is relevant to all manner of tasks, and not just to computer programming. The combination of concision and precision, the sine qua non of software design, makes APS stand out from other accounts of problem solving.

\section{- Focus on method.}

The problem-oriented nature of the module means that its focus is truly on method rather than knowledge. For example, it would be stupid to suppose that being able to memorise a knight's circuit of a chessboard will ever be a useful skill! But a study of how the problem is analysed and broken down into simpler problems, and how these simpler problems are solved is very frutiful because the same skills can be applied elsewhere (are "transferrable" in current jargon). Unfortunately, algorithm design is often presented in a knowledge-based fashion, reducing it to a specialist skill. It is much harder to instil good method, but much more worthwhile. APS tries to articulate sound problem-solving skills, even though these may be informal and imprecise.

Because of time limitations, the module does not go as far as I would like to take it. This year, for the first time, I have been given the opportunity to integrate the problem-oriented approach of APS with an introduction to calculational mathematics. I hope to be able to report on this endeavour in the near future.

On a longer timescale, I believe that there is a real scope for a revolution in the way that mathematics - the art of effective reasoning - is taught and practised, both at school and university, a revolution driven by the lessons learnt from correct-by-construction programming techniques. This is my "grand challenge" for research and education in formal methods and the science of computing.

\section{REFERENCES}

[1] Roland Backhouse. Program Construction. Calculating Implementations From Specifications. John Wiley \& Sons, 2003.

[2] Roland Backhouse and Maarten Fokkinga. The associativity of equivalence and the Towers of Hanoi Problem. Information Processing Letters, 77:71-76, 2001.

[3] Elwyn R. Berlekamp, John H. Conway, and Richard K. Guy. Winning Ways, volume I and II. Academic Press, 1982.

[4] Charles L. Bouton. Nim, a game with a complete mathematical theory. Ann. of Math., Princeton, 3(2):35-39, 1901-02.

[5] Edsger W. Dijkstra. Guarded commands, nondeterminacy and formal derivation of programs. Communications of the ACM, 18:453-457, 1975.

[6] Edsger W. Dijkstra. EWD1083: The balance and the coins. http://www.cs .utexas . edu/users/EWD/ewd10xx/EWD1083. PDF, September 1990.

[7] Edsger W. Dijkstra. EWD1260: The marked coins and the scale. http://www.cs .utexas. edu/users/EWD/ewd12xx/EWD1260. PDF, March 1997.

[8] David Gries and Fred B. Schneider. A Logical Approach to Discrete Math. Springer-Verlag, 1993.

[9] P.M. Grundy and C.A.B. Smith. Disjunctive games with the last player losing. Proc. Cambridge Philos. Soc., 52:527-533, 1956.

[10] lan Stewart. The Magical Maze. Weidenfield and Nicolson, London, 1997.

[11] A.J.M. van Gasteren. On the Shape of Mathematical Arguments. Number 445 in LNCS. Springer-Verlag, 1990. 